\title{
(อ) OPEN ACCESS \\ Fifteen-minute consultation: Initial management of suspected acute leukaemia by non-specialists
}

\author{
Sam Behjati, ${ }^{1,2}$ Amy Ruffle, ${ }^{2,3}$ Anne Kelly, ${ }^{2}$ Emmy Dickens ${ }^{2}$
}

\begin{abstract}
Department of Paediatrics, University of Cambridge, Cambridge, UK

${ }^{2}$ Department of Paediatric Oncology and Haematology, Addenbrooke's Hospital, Cambridge, UK

${ }^{3}$ Department of Haematology and Oncology, Great Ormond Street Hospital for Children NHS Foundation Trust, London, UK
\end{abstract}

\section{Correspondence to}

Dr Sam Behjati, University of Cambridge, Cambridge CB2

1TN, UK; sam.behjati@gmail. com

SB and AR contributed equally.

SB and AR were joint first authors.

Received 11 November 2018 Revised 7 April 2019

Accepted 14 April 2019

Published Online First

5 July 2019

\section{Check for updates}

(c) Author(s) (or their employer(s)) 2020. Re-use permitted under CC BY. Published by BMJ.

To cite: Behjati S, Ruffle A, Kelly $\mathrm{A}$, et al. Arch Dis Child Educ Pract Ed 2020;105:66-70.

\begin{abstract}
Leukaemia is the most common cancer of childhood. Most children with a new diagnosis of leukaemia are clinically stable at initial presentation. However, there are a number of life-threatening complications that have to be considered and monitored for. These complications include sepsis, tumour lysis syndrome, mediastinal masses, bleeding and pain. The aim of this article is to equip the general paediatrician with a framework for managing children with suspected leukaemia, prior to transfer to the primary treatment centre. The presentation, diagnosis and definitive treatment of acute leukaemia is not in the remit of this article.
\end{abstract}

\section{INTRODUCTION}

All paediatricians will at some point care for a child who presents with suspected leukaemia. A typical-sized district general hospital (DGH) can expect approximately three children to be diagnosed with leukaemia each year, with around 420 new cases diagnosed annually in England. ${ }^{1}$

\section{INVESTIGATIONS}

While most children presenting with a new diagnosis of acute leukaemia remain clinically stable, occasionally children can become critically unwell. Early death in leukaemia is rare, although young age, particularly those under 2 years, high white cell count (more than $100 \times 10^{9}$ cells $/ \mathrm{L}$ ) and acute myeloid leukaemia (AML) are established risk factors.

It is important to discuss suspected cases of new leukaemia with the primary treatment centre (PTC) early while arranging investigations (table 1) and starting appropriate treatment (table 2). The overall aim of the initial management is to anticipate and, where required, manage complications prior to transfer.
It is useful to send a stained blood film and an additional EDTA sample to the PTC either before transfer or along with the child. Early availability of these samples at the PCT will greatly facilitate the diagnostic work-up, allowing blood film microscopy and flow cytometric analysis of peripheral blood. In the past, it was important to send a thiopurine methyltransferase (TPMT) level before red cell transfusion to predict the child's ability to metabolise thiopurine drugs in order to determine dosing of 6-mercaptopurine. However, TPMT enzyme levels have now been superseded by testing for the TPMT genotype, which is not affected by transfusion and will be arranged by the PTC.

Some PTCs may also request testing for glucose-6-phosphate dehydrogenase deficiency given the risk of precipitating a crisis in undiagnosed children if rasburicase is administered.

\section{COMPLICATIONS AND MANAGEMENT Infection}

As fewer children die from their disease, infection has become one of the leading causes of death in children with leukaemia. Sepsis was responsible for over $30 \%$ of all deaths in children in the most recently completed UK childhood ALL trial, UKALL 2003, and resulted in almost as many deaths as acute lymphoblastic leukaemia itself. $^{23}$

At diagnosis, children with acute leukaemia are immunosuppressed despite not having started chemotherapy, due to a combination of bone marrow infiltration and other factors that interfere with immune cellular function. Thus, children with suspected leukaemia should be managed as per neutropaenic sepsis guidelines, irrespective of their neutrophil count. If there is any history of fever or other indicators of infection, take blood cultures and start intravenous antibiotics 


\section{Table 1 Initial mandatory investigations}

\begin{tabular}{|c|c|}
\hline Test & Indication \\
\hline Chest X-ray & To identify a mediastinal mass, consolidation due to infection or alveolar infiltrates suggestive of leucostasis. \\
\hline Serial full blood counts & Assessment of white cell count and rate of change, anaemia and thrombocytopaenia. \\
\hline $\begin{array}{l}\text { Serial urea, electrolytes, creatinine, } \\
\text { bone profile and uric acid }\end{array}$ & $\begin{array}{l}\text { Tumour lysis syndrome is characterised by hyperuricaemia hyperphosphataemia, hyperkalaemia and } \\
\text { hypocalcaemia leading to renal injury. }\end{array}$ \\
\hline Blood cultures (if indicated) & Prior to staring intravenous antibiotics, blood cultures should be taken. \\
\hline Group and save & In anticipation of potential need for blood products \\
\hline $\begin{array}{l}\text { Clotting (prothrombin time, activated } \\
\text { partial thromboplastin time and } \\
\text { fibrinogen) }\end{array}$ & Disseminated intravascular coagulation is occasionally seen in association with acute leukaemia. \\
\hline Blood film & $\begin{array}{l}\text { Can usually be examined by the on-call haematologist at the referring hospital, with a second film made and } \\
\text { sent with the child to the PTC. }\end{array}$ \\
\hline Additional EDTA sample & $\begin{array}{l}\text { Peripheral blood can be sent to the PTC for immunophenotyping, which may be sufficient to confirm or } \\
\text { refute the diagnosis of leukaemia. }\end{array}$ \\
\hline Viral serology & $\begin{array}{l}\text { Pretransfusion viral serological tests (especially VZV) are required for every child with a new diagnosis of } \\
\text { cancer. Typically a virology screen includes VZV, HSV1, HSV2, EBV, measles, hepatitis B and hepatitis C. }\end{array}$ \\
\hline
\end{tabular}

PTC, primary treatment centre.

according to local policies. If there is clinical evidence of sepsis, manage this as you would in any child, aggressively within 'the golden hour'. ${ }^{4}$

\section{Tumour lysis syndrome (TLS)}

TLS is a metabolic disturbance resulting from the breakdown of tumour cells. Cellular lysis causes the release and degradation of intracellular contents including nucleic acids, which have the potential to overwhelm the body's normal homeostatic mechanisms. ${ }^{5}$ The resulting biochemical abnormalities include hyperuricaemia, hyperkalaemia and hyperphosphataemia with secondary hypocalcaemia. Uric acid precipitates within renal tubules; phosphate crystallises with calcium within soft tissues, including the kidney. Together these can result in acute kidney injury, reducing the body's ability to excrete electrolytes, which sets up a spiral of worsening metabolic derangement and kidney failure.

It therefore follows that children at greater risk of TLS are those with a large volume of disease. These are children with a high white cell count (more than
$50 \times 10^{9} \mathrm{cells} / \mathrm{L}$ ) or 'bulky' disease such as significant hepatosplenomegaly, lymphadenopathy or a mediastinal mass. In addition, pre-existing impaired renal function puts children at higher risk of TLS. Although TLS is most frequently seen following administration of steroids and chemotherapy agents, it can occur spontaneously.

Management of TLS is largely anticipatory to prevent its development. In acute leukaemia, some children with low count disease and without bulky disease can be managed with maintenance fluids, which can be oral or intravenous. Others at higher risk will require hyperhydration even prior to starting chemotherapy, with intravenous fluids of at least $2.5 \mathrm{~L} / \mathrm{m}^{2} /$ day. ${ }^{6}$ With the guidance of the PTC, the fluid rate may be increased if there is any evidence or significant risk of TLS. Potassium should not be added to fluids due to the risk of hyperkalaemia, even if potassium levels are very low. Children should be monitored for fluid overload, particularly children with severe anaemia and infants. In addition to records of fluid input and output, daily

Table 2 Management points to consider

\begin{tabular}{|c|c|}
\hline Intervention & Indications \\
\hline Oxygen & $\begin{array}{l}\text { Hypoxia and/or respiratory distress that might indicate severe anaemia, infection, leucostasis within the lung vasculature } \\
\text { or a mediastinal mass. }\end{array}$ \\
\hline Antibiotics & $\begin{array}{l}\text { Broad-spectrum antibiotics following local guidelines for neutropaenic sepsis should be started if there are any concerns } \\
\text { regarding infection irrespective of neutrophil count. }\end{array}$ \\
\hline Analgesia & Paracetamol \pm oral morphine. Non-steroidal anti-inflammatory drugs are best avoided in thrombocytopaenia. \\
\hline $\begin{array}{l}\text { Hyperhydration } \\
\text { Allopurinol or } \\
\text { rasburicase }\end{array}$ & $\begin{array}{l}\text { For prevention of tumour lysis syndrome following discussion with the PTC. However, transfer to the PTC should not be } \\
\text { delayed if there is difficulty in obtaining these drugs. }\end{array}$ \\
\hline Blood products & $\begin{array}{l}\text { Platelets, fresh frozen plasma and/or cryoprecipitate transfusion may be required to treat bleeding. Red cell transfusion } \\
\text { should be considered carefully particularly in the context of a high or rising white cell count because the further increase } \\
\text { in viscosity from packed red cell transfusion can precipitate complications of hyperleucostasis. }\end{array}$ \\
\hline
\end{tabular}

PTC, primary treatment centre. 


\section{Box 1 The first cannula}

Children undergoing cancer treatment often become needle phobic. For the first few days, and in many cases, the first month, treatment will be given through peripheral intravenous access. Therefore, it is important to aim to minimise trauma from the very first episode of cannulation. If your patient is stable enough, take your time, use a local anaesthetic cream and distraction techniques. Get all the blood bottles ready and calculate the total volume of blood required. If possible, insert a $22 \mathrm{G}$ or $20 \mathrm{G}$ cannula with the aim of using it to bleed back for regular tumour lysis blood tests. Try to avoid dorsal hand and foot veins, which may later be needed for vincristine administration. It is also preferable for the practitioner doing the cannulation to be the person most likely to be successful on the first attempt.

or twice daily weight measurements are very useful in assessing fluid balance, especially in infants. Frusemide can be used to maintain diuresis although it should be used with caution if there is risk of leucostasis.

Allopurinol should be started in children where the clinical suspicion of acute leukaemia is high. ${ }^{6}$ It is a xanthine oxidase inhibitor, which prevents the formation of uric acid. In children either at high risk of developing TLS or with biochemical signs of the syndrome, rasburicase may be used as an alternative to allopurinol. This recombinant urate oxidase actively degrades uric acid and is a more potent therapy against TLS. However, it should be avoided in children with glucose-6-phosphate dehydrogenase deficiency as this can precipitate a haemolytic crisis. Recommended doses for allopurinol and rasburicase may differ between leukaemia treatment protocols (eg, UKALL 2011 ${ }^{6}$ ) and pharmacopoeias such as the British National Formulary (BNF). Therefore, seek advice from your PCT on current drug doses and treatment schedules for both drugs.

Initially, TLS is an asymptomatic biochemical abnormality and your PTC will guide how frequently monitoring bloods should be taken, but this may be as often as every 4 hours in high-risk cases. Note that in very high count leukaemia, cells may haemolyse in the specimen bottle en route to the laboratory, leading to false electrolyte readings. It is therefore advisable to perform paired gas machine and laboratory electrolyte measurement when the white cell count is high. It is important to discuss any abnormalities, including both elevated and low electrolytes, early and before making corrections as these may show an evolving picture. Very occasionally, children with severe TLS will require haemofiltration.

\section{Mediastinal mass}

Children with leukaemia, particularly with $\mathrm{T}$ ell disease, may have mediastinal lymphadenopathy, which can cause airway obstruction. Signs and symptoms of an obstructive mediastinal mass include wheezing, stridor, orthopnoea (specifically ask about this) and signs of superior vena cava obstruction (facial swelling and prominent veins). Should a child have any symptoms or signs of a mediastinal mass, it is important to consider an early anaesthetic assessment and organise urgent transfer to the PTC. Children should be nursed sitting upright. Lying flat, even for the short duration of a CT chest, may precipitate airway obstruction.

General anaesthetic and sedation with drugs such as chloral hydrate or midazolam should be avoided if there is any suspicion of a mediastinal mass, due to the risk of precipitating airway compromise and cardiovascular instability, even in a previously asymptomatic child. This is because these drugs cause a reduction in tone in both airway and vascular structures. ${ }^{7}$

\section{Hyperleucocytosis and leucostasis}

Hyperleucocytosis is defined as a white cell count greater than $50 \times 10^{9}$ cells/L. However, this threshold is arbitrary and complications from hyperleucocytosis can be seen at lower counts, especially in AML. ${ }^{8}$ Hyperleucocytosis significantly increases the risk of morbidity and mortality. For instance, a recent case series reported a $30 \%$ mortality within 2 weeks of diagnosis in children with AML who had a white cell count of greater than $200 \times 10^{9}$ cells/L. ${ }^{9}$ Seeking the opinion of your local consultant haematologist on the blood film appearance may help you to identify AML early and thus identify the increased risk of complications from hyperleucocytosis.

Leucostasis is the process of increased blood viscosity, poorly deformable blasts and cytokine-induced activation of the endothelium resulting in vascular obstruction and tissue hypoxia. If this process occurs in the lungs, shortness of breath, coughing and hypoxia may ensue. A plain chest radiograph may show diffuse interstitial or alveolar infiltrates. Leucostasis can also affect cerebral vessels and cause haemorrhagic or ischaemic events leading to symptoms of confusion, dizziness, tinnitus and headaches, as well as focal neurological deficits. Other manifestations of leucostasis include priapism, limb ischaemia, renal vein thrombosis and retinal haemorrhage. ${ }^{8}$

Management includes oxygen, hyperhydration and urgent discussion and transfer to the PTC. Definitive treatment is that of the underlying leukaemia, and it is important this is not delayed by other interventions. However, within an intensive care setting, apheresis or an exchange transfusion may occasionally be instigated.

\section{Coagulopathy}

Serious bleeding in acute leukaemia is rare with only $3 \%$ of children showing evidence of coagulopathy at diagnosis. ${ }^{10}$ There is a greater risk of serious bleeding at presentation in some children, including those with acute myeloid leukaemia (AML) and hyperleucocytosis. There is a rare subtype of myeloid leukaemia, 


\section{Box 2 Communicating the diagnosis of leukaemia}

- Not all families are aware that leukaemia is a malignant disease, and it may help to describe the condition as a 'cancer of the blood'.

- It is unlikely that a definitive diagnosis of leukaemia will be made prior to transfer. Therefore, terms such as 'suspected leukaemia' or 'likely leukaemia' may be useful and avoid committing to any specific subtype of leukaemia solely on the basis of a blood film.

- Parents may ask about prognosis, and it would be reasonable to tell them that it is highly likely that the disease will be treatable but that more specific information can only be given after the results of further tests.

- Transfer to a children's oncology ward with an uncertain diagnosis can be frightening, both for parents and children. This can be helped with sensitive preparation.

acute promyelocytic leukaemia, which requires aggressive correction of coagulopathy to prevent life-threatening bleeds at presentation. The Nordic group reported eight deaths (14\%) from intracranial haemorrhage and/or disseminated intravascular coagulation, out of a total of 57 patients with AML and a WCC more than $200 \times 10^{9}$ cells $/$ L. ${ }^{9}$ By contrast, significant coagulopathy is extremely uncommon in acute lymphoblastic leukaemia (ALL) with only two cases of intracranial haemorrhage seen in over 2500 children in the UKALL 2003 trial. $^{11}$

Coagulation should always be checked at diagnosis, ${ }^{12}$ although the correlation between serious haemorrhage, coagulopathy or the use of prophylactic plasma is questionable. ${ }^{13}$ When considering correction of abnormal clotting results in the absence of bleeding, it is important to consider the potential risks of administering plasma components. Discuss any abnormal results with your PTC who will provide patientspecific guidance.

\section{Blood product support}

Although serious bleeding is rare, minor bleeding is much more common, affecting 38\% of children with ALL at diagnosis. ${ }^{14}$ This is related to thrombocytopaenia and therefore affects predominantly the mucosa and skin. National guidelines would advise transfusing platelets if platelet counts are less than $10 \times 10^{9}$ platelets/L. Raising this threshold to $20 \times 10^{9}$ platelets $/ \mathrm{L}$ in feverish children routinely is no longer recommended. ${ }^{15}$ In the presence of significant bleeding transfusion should be based on the clinical situation rather than the platelet count.

At presentation, many children will be anaemic, but red cell transfusions may not be required. In hyperleucocytosis, red cell transfusion should be avoided due to the risk of complications of leucostasis such as TLS or stroke. Therefore, the conventional transfusion thresholds for children undergoing chemotherapy (below $\left.70-80 \mathrm{~g} / \mathrm{L}^{15}\right)$, do not necessarily apply at presentation, and discussion with the PCT is advised before giving red cells. When transfusion is required due to severe anaemia, in the context of hyperleucocytosis, smaller volumes are often used, such as $5 \mathrm{~mL} / \mathrm{kg}$ over 4 hours, in parallel with hyperhydration.

At diagnosis, UK national guidelines ${ }^{16}$ do not recommend any special blood product requirements as all blood products are now hepatitis $\mathrm{E}$ negative and leucodepleted. However, your own PTC may have local guidelines regarding cytomegalovirus-screened blood products and also suggest viral serology testing before transfusion. There is no specific evidence to guide the optimum haemoglobin transfusion threshold, although current practice would suggest that a threshold between $70 \mathrm{~g} / \mathrm{L}$ and $80 \mathrm{~g} / \mathrm{L}$ may be reasonable.

\section{Pain}

Pain is surprisingly common at presentation in leukaemia with $43 \%$ of children having limb pain at presentation ${ }^{14}$ and this may be under-recognised. Surrogate markers of pain can be useful, such as not using limbs and being clingy or unsettled. Nonsteroidal anti-inflammatory drugs are best avoided due to their effects on platelet function in a child who is already at risk of thrombocytopaenia due to their underlying disease. In this context, oral morphine sulfate may be useful. Attempts should also be made to minimise procedural pain (box 1).

\section{COMMUNICATION}

It is essential to tell the family that a diagnosis of leukaemia is suspected prior to transfer to the PTC. Families need to be prepared that they will be transferred to an oncology ward, which can identified as such from signs, posters and patients. Similarly, children should be given age appropriate information. While a detailed discussion of how to break bad news is beyond the scope of this article, there are some specific points we would like to highlight about communicating a diagnosis of suspected leukaemia (box 2).

\section{Clinical bottom line}

- Communicate early with your primary treatment centre.

- Most children presenting with acute leukaemia are clinically stable yet have the potential to deteriorate and require intensive clinical care.

> Monitoring for complications should include infection, tumour lysis syndrome, hyperleucocytosis and coagulopathy.

- Ensure appropriate analgesia; some children will require oral morphine for adequate pain relief.

- Parents should be aware that you suspect leukaemia prior to transfer. 


\section{TRANSFER}

It is important to consider and discuss with your PTC an appropriate timing for transfer, and these conversations should include senior decision makers. There are some children who require urgent transfer, such as those with a very high white cell count, mediastinal mass or clinical instability. Many children who are clinically stable and have low count disease can be safely managed in a DGH overnight, or even for several days, if there is no bed immediately available in the nearest PTC. These children need to be monitored closely with regular observations, clinical assessment and blood tests and require regular discussion with the PTC.

Acknowledgements We would like to thank Dr Kristina Marshall for reviewing the manuscript.

Contributors SB conceived of the paper. AR and SB were both co-authors on this paper. AR did the literature search. ED and AK contributed expert views. All authors have contributed to editing successive drafts and agreed the content of the final article.

Funding This study was funded by Wellcome Trust (Grant 110104/Z/15/Z).

Competing interests None declared.

Patient consent for publication Not required.

Provenance and peer review Commissioned; externally peer reviewed.

Open access This is an open access article distributed in accordance with the Creative Commons Attribution 4.0 Unported (CC BY 4.0) license, which permits others to copy, redistribute, remix, transform and build upon this work for any purpose, provided the original work is properly cited, a link to the licence is given, and indication of whether changes were made. See: https:// creativecommons.org/licenses/by/4.0/.

\section{REFERENCES}

1 England PH. Childhood cancer registration in England: 2015 to 2016, 2016.

2 Blanco E, Beyene J, Maloney AM, et al. Non-relapse mortality in pediatric acute lymphoblastic leukemia: a systematic review and meta-analysis. Leuk Lymphoma 2012;53:878-85.

3 O'Connor D, Bate J, Wade R, et al. Infection-related mortality in children with acute lymphoblastic leukemia: an analysis of infectious deaths on UKALL2003. Blood 2014;124:1056-61.
4 Anon. Sepsis: recognition, diagnosis and early management: (C) NICE (2017) Sepsis: recognition, diagnosis and early management. BJU Int 2018;121:497-514.

5 Jones GL, Will A, Jackson GH, et al. Guidelines for the management of tumour lysis syndrome in adults and children with haematological malignancies on behalf of the British Committee for Standards in Haematology. Br J Haematol 2015;169:661-71.

6 UKALL2011 Trial: United Kingdom National Randomised Trial For Children and Young Adults with Acute Lymphoblastic Leukaemia and Lymphoma, 2011.

7 Hammer GB. Anaesthetic management for the child with a mediastinal mass. Paediatr Anaesth 2004;14:95-7.

8 Ganzel C, Becker J, Mintz PD, et al. Hyperleukocytosis, leukostasis and leukapheresis: practice management. Blood Rev 2012;26:117-22.

9 Zeller B, et al. Hyperleucocytosis in paediatric acute myeloid leukaemia - the challenge of white blood cell counts above 200 $\times$ 10. Br J Haematol 2017;178:448-56.

10 Ribeiro RC, Pui CH. The clinical and biological correlates of coagulopathy in children with acute leukemia. J Clin Oncol 1986;4:1212-8.

11 Astwood E, Vora A. Personal practice: how we manage the risk of bleeding and thrombosis in children and young adults with acute lymphoblastic leukaemia. Br J Haematol 2011;152:50511.

12 Sibson KR, Biss TT, Furness CL, et al. BSH Guideline: management of thrombotic and haemostatic issues in paediatric malignancy. Br J Haematol 2018;180:511-25.

13 Sutor AH, Mall V, Thomas KB. Bleeding and thrombosis in children with acute lymphoblastic leukaemia, treated according to the ALL-BFM-90 protocol. Klin Padiatr 1999;211:201-4.

14 Clarke RT, Van den Bruel A, Bankhead C, et al. Clinical presentation of childhood leukaemia: a systematic review and meta-analysis. Arch Dis Child 2016;101:894-901.

15 National Institute for Health and Care Excellence. Blood transfusion (NICE Guideline 24). 2015 https://www.nice.org. uk/guidance/ng24 (Accessed 27 Feb 2019).

16 New HV, Berryman J, Bolton-Maggs PH, et al. Guidelines on transfusion for fetuses, neonates and older children. $\mathrm{Br} \mathrm{J}$ Haematol 2016;175:784-828. 